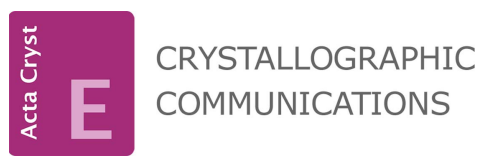

ISSN 2056-9890

Received 26 September 2015

Accepted 29 October 2015

Edited by G. Smith, Queensland University of Technology, Australia

Keywords: crystal structure; organic-inorganic hybrid; piperidinium salts; hydrogen-bonding

CCDC reference: 1434209

Supporting information: this article has supporting information at journals.iucr.org/e

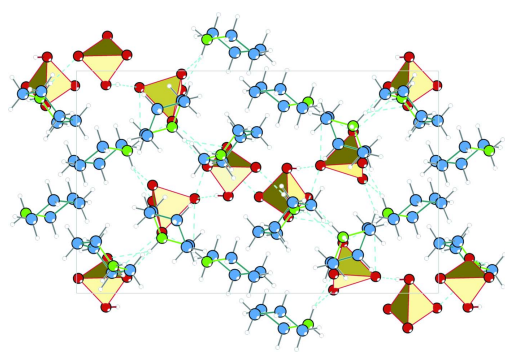

OPEN $\odot$ ACCESS

\section{Crystal structure of tris(piperidinium) hydrogen sulfate sulfate}

\author{
Tamara J. Lukianova, ${ }^{*}$ Vasyl Kinzhybalo and Adam Pietraszko
}

Institute of Low Temperature and Structure Research, Polish Academy of Sciences, Okolna str. 2, PO Box 1410, 50-950 Wroclaw, Poland. *Correspondence e-mail: t.lukianova@int.pan.wroc.pl

In the title molecular salt, $3 \mathrm{C}_{5} \mathrm{H}_{12} \mathrm{~N}^{+} \cdot \mathrm{HSO}_{4}{ }^{-} \cdot \mathrm{SO}_{4}{ }^{2-}$, each cation adopts a chair conformation. In the crystal, the hydrogen sulfate ion is connected to the sulfate ion by a strong $\mathrm{O}-\mathrm{H} \cdots \mathrm{O}$ hydrogen bond. The packing also features a number of $\mathrm{N}-\mathrm{H} \cdots \mathrm{O}$ hydrogen bonds, which lead to a three-dimensional network structure. The hydrogen sulfate anion accepts four hydrogen bonds from two cations, whereas the sulfate ion, as an acceptor, binds to five separate piperidinium cations, forming seven hydrogen bonds.

\section{Chemical context}

Hydrogen bonding is a powerful and versatile tool commonly used in crystal engineering to design, combine and organize individual organic molecules in solids, thus creating new materials with tunable physical properties. Simple organicinorganic salts seem to be good candidates for this purpose because of the flexibility of their special structural features such as polarity and their promising potential applications in chemistry. Not of less importance would be the use of inorganic oxyanions, which are very attractive as inorganic building blocks due to their shapes and diverse reactivity in aqueous solutions. In recent years, sulfates and hydrogen sulfates of organic bases have found applications as ionic liquids (George et al., 2015). Therefore, the results of a structural study on a new molecular salt obtained from piperidine and sulfuric acid are reported here.<smiles>C1CC[NH2+]CC1</smiles><smiles>O=S(=O)([O-])O</smiles><smiles>O=S(=O)([O-])[O-]</smiles>

\section{Structural commentary}

In the title compound, $3 \mathrm{C}_{5} \mathrm{H}_{12} \mathrm{~N}^{+} \cdot \mathrm{HSO}_{4}{ }^{-} \cdot \mathrm{SO}_{4}{ }^{2-}$, (I), the asymmetric unit comprises three independent protonated piperidinium cations, one hydrogen sulfate anion and one sulfate anion (Fig. 1). The geometries of the three cations are similar, possessing chair conformations. The $\mathrm{N}-\mathrm{C}$ and $\mathrm{C}-\mathrm{C}$ bond lengths are in the ranges 1.489 (2)-1.4978 (19) $\AA$ and 1.518 (2) -1.530 (2) $\AA$, respectively. The $\mathrm{C}-\mathrm{C}-\mathrm{C}, \mathrm{C}-\mathrm{C}-\mathrm{N}$ and $\mathrm{C}-\mathrm{N}-\mathrm{C}$ angles are in the ranges 109.69 (13)-111.42 (13), $109.20(12)-110.29$ (12) and $112.01(11)-112.30(12)^{\circ}$, respectively. These values are in good agreement with those reported in the literature (Lee \& Harrison, 2003). Within the cationanion unit, the $\mathrm{N}$ atoms of the three piperidinium cations are 


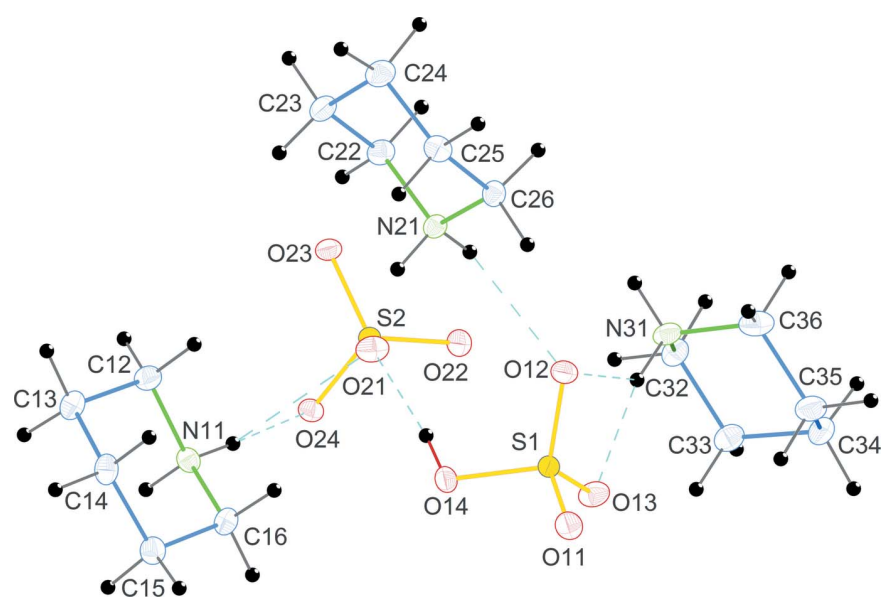

Figure 1

The asymmetric unit of (I), showing the atom-numbering scheme. Displacement ellipsoids are drawn at the $50 \%$ probability level. Hydrogen bonds are denoted by cyan dashed lines.

connected to the $\mathrm{O}$ atom acceptors of the $\mathrm{HSO}_{4}{ }^{-}$(O11-O14) and $\mathrm{SO}_{4}{ }^{2-}(\mathrm{O} 21-\mathrm{O} 24)$ anions by five $\mathrm{N}-\mathrm{H} \cdots \mathrm{O}$ hydrogen bonds (Table 1). The two anions are linked via a short O14H14. . O21 hydrogen bond [2.5603 (16) Å], Figs. 1 and 2.

\section{Supramolecular features}

The crystal structure of (I) features $\mathrm{N}-\mathrm{H} \cdots \mathrm{O}$ and $\mathrm{O}-\mathrm{H} \cdots \mathrm{O}$ hydrogen bonds (Table 1, Fig. 1). The $\mathrm{N}$ atoms of the piperidinium cations are involved in hydrogen-bond formation, as donors with oxygen atoms of the sulfate and hydrogen sulfate anions. The sulfate-bound $\mathrm{O}$ atoms, which act as acceptors, link the organic molecules through rather strong hydrogen bonds, forming a two-dimensional network of hydrogen bonds giving rise to layers parallel to (100). The hydrogen sulfate ion accepts four hydrogen bonds from three cations, whereas the sulfate ion, as an acceptor, binds to five piperidinium ions, forming seven hydrogen bonds in the overall three-dimensional structure (Fig. 3).

\section{Database survey}

Crystal structures of piperidinium cations with counter-anions such as hydrogen sulfide, arsenate and violurate (Smail \&

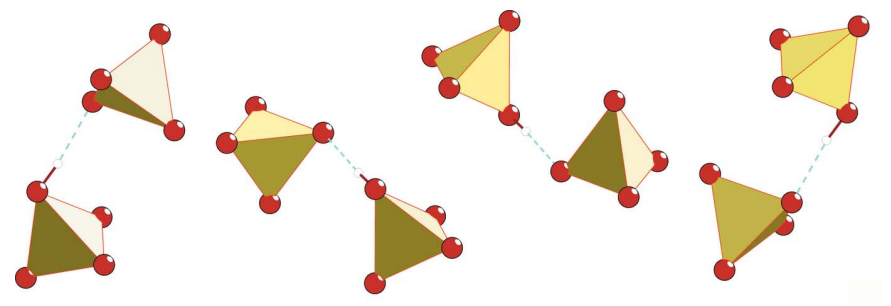

Figure 2

The fragments of $\mathrm{HS}_{2} \mathrm{O}_{8}{ }^{3-}$ anion pairs, formed from $\mathrm{HSO}_{4}{ }^{-}$and $\mathrm{SO}_{4}{ }^{2-}$ anions via strong $\mathrm{O}-\mathrm{H} \cdots \mathrm{O}$ hydrogen bonds (cyan dashed lines).
Table 1

Hydrogen-bond geometry $\left(\AA{ }^{\circ}\right)$.

\begin{tabular}{lllll}
\hline$D-\mathrm{H} \cdots A$ & $D-\mathrm{H}$ & $\mathrm{H} \cdots A$ & $D \cdots A$ & $D-\mathrm{H} \cdots A$ \\
\hline $\mathrm{O} 14-\mathrm{H} 14 \cdots \mathrm{O} 21$ & 0.84 & 1.72 & $2.5603(16)$ & 173 \\
$\mathrm{~N} 21-\mathrm{H} 21 A \cdots \mathrm{O} 11^{\mathrm{i}}$ & 0.91 & 1.93 & $2.8226(18)$ & 166 \\
$\mathrm{~N} 21-\mathrm{H} 21 B \cdots \mathrm{O} 12$ & 0.91 & 2.32 & $2.9096(19)$ & 122 \\
$\mathrm{~N} 21-\mathrm{H} 21 B \cdots \mathrm{O} 24^{\text {ii }}$ & 0.91 & 2.47 & $3.0964(18)$ & 127 \\
$\mathrm{~N} 11-\mathrm{H} 11 A \cdots \mathrm{O} 21$ & 0.91 & 2.59 & $3.201(2)$ & 126 \\
$\mathrm{~N} 11-\mathrm{H} 11 A \cdots \mathrm{O} 24$ & 0.91 & 1.89 & $2.7904(17)$ & 171 \\
$\mathrm{~N} 11-\mathrm{H} 11 B \cdots \mathrm{O} 22^{\text {iii }}$ & 0.91 & 2.47 & $3.0474(18)$ & 122 \\
$\mathrm{~N} 11-\mathrm{H} 11 B \cdots \mathrm{O} 23^{\text {iii }}$ & 0.91 & 1.92 & $2.8039(18)$ & 164 \\
$\mathrm{~N} 31-\mathrm{H} 31 A \cdots \mathrm{O} 12$ & 0.91 & 2.41 & $3.0245(18)$ & 125 \\
$\mathrm{~N} 31-\mathrm{H} 31 A \cdots \mathrm{O} 13$ & 0.91 & 1.93 & $2.8240(18)$ & 167 \\
$\mathrm{~N} 31-\mathrm{H} 31 B \cdots \mathrm{O} 24^{\text {ii }}$ & 0.91 & 1.89 & $2.7978(19)$ & 172 \\
\hline
\end{tabular}

Symmetry codes: (i) $-x+1,-y+1,-z+1$; (ii) $x,-y+\frac{3}{2}, z+\frac{1}{2}$; (iii) $x,-y+\frac{3}{2}, z-\frac{1}{2}$.

Sheldrick, 1973; Lee \& Harrison, 2003; Kolev et al., 2009) and other mixed compounds (Banerjee \& Murugavel, 2004; Mohammadnezhad et al., 2008; Xu et al., 2009; Anderson et al., 2011; Hoque \& Das, 2014) have been reported.

\section{Synthesis and crystallization}

The title compound was prepared by the reaction between $3 \mathrm{ml}(0.03 \mathrm{~mol})$ of piperidine (Aldrich, ReagentPlus, $99 \%)$ and

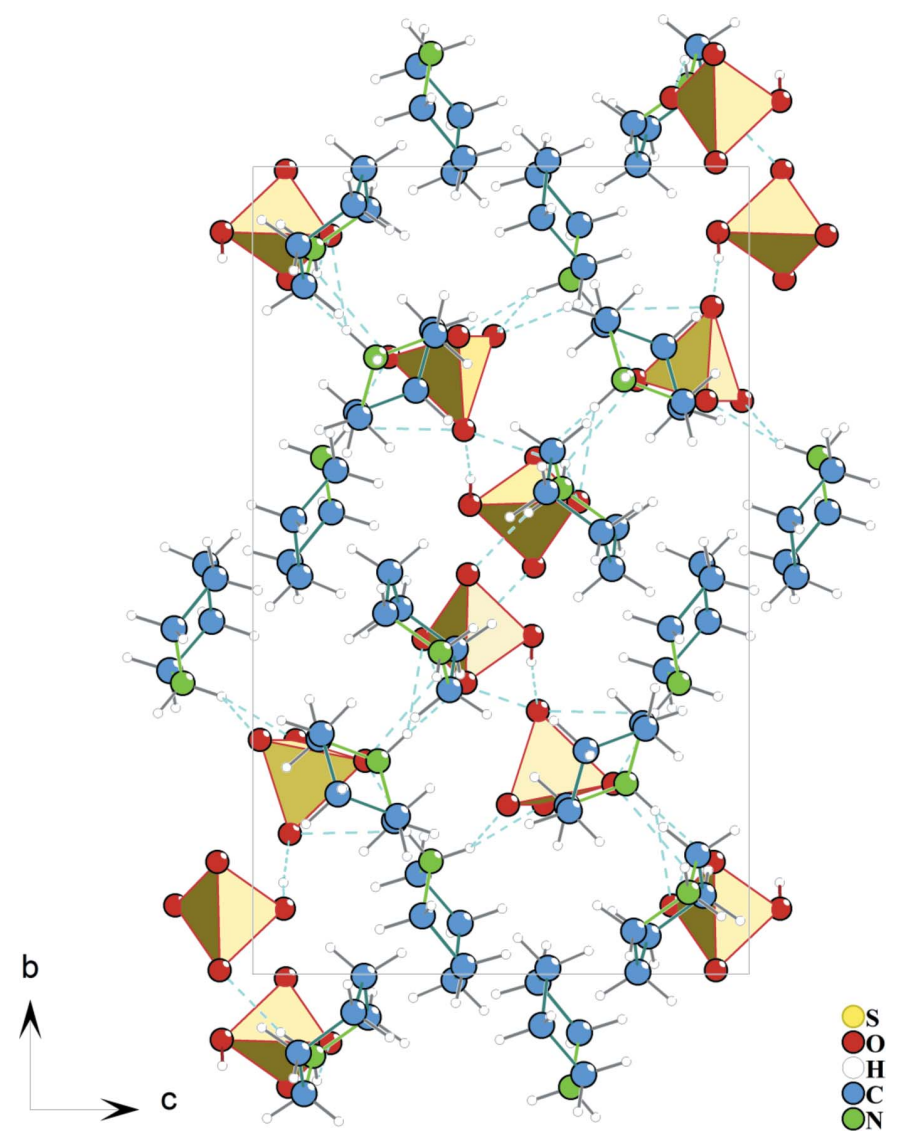

Figure 3

Projection of the crystal structure of (I) on the (100) plane. Hydrogen bonds are denoted by cyan dashed lines. 
$3.1 \mathrm{ml}(0.012 \mathrm{~mol})$ of $30 \%$ aqueous sulfuric acid solution. The reaction mixture was continuously stirred for 15 minutes at $323 \mathrm{~K}$ and then allowed to cool down to room temperature. The final $\mathrm{pH}$ value was 2 . The mixture was kept at room temperature over a period of several months, after which it was cooled in a refrigerator $(T \simeq 278 \mathrm{~K})$, giving colourless crystals of the title compound after a few weeks.

\section{Refinement}

Crystal data, data collection and structure refinement details are summarized in Table 2. The positions of hydrogen atoms of the amines and the hydrogen sulfate anion were initially located in difference Fourier maps but were subsequently allowed to ride in the refinement with $\mathrm{O}-\mathrm{H}=0.84$ and $\mathrm{N}-\mathrm{H}$ $=0.91 \AA$ and with $U_{\text {iso }}(\mathrm{H})=1.2 U_{\text {eq }}(\mathrm{N})$ or $1.5 U_{\text {eq }}(\mathrm{O})$. The $\mathrm{H}$ atom of the hydrogen sulfate anion was refined with the SHELX AFIX 147 instruction. Piperidinium C-bound $\mathrm{H}$ atoms were placed in geometrically idealized positions and also allowed to ride, with $\mathrm{C}-\mathrm{H}=0.99 \AA$ and $U_{\text {iso }}(\mathrm{H})=$ $1.2 U_{\text {eq }}(\mathrm{C})$.

\section{Acknowledgements}

The authors thank Dr M. Marchewka for providing chemicals for this synthesis. This research was supported by an ILT\&SR PAS grant for young scientists and PhD students, funded by the Ministry of Science and Higher Education of Poland.

\section{References}

Anderson, K. M., Goeta, A. E., Martin, J. E., Mason, S. A., McIntyre, G. J., Sansam, B. C. R., Wilkinson, C. \& Steed, J. W. (2011). Cryst. Growth Des. 11, 4904-4919.

Banerjee, S. \& Murugavel, R. (2004). Cryst. Growth Des. 4, 545-552.

Brandenburg, K. (1997). DIAMOND. Crystal Impact GbR, Bonn, Germany.

Clark, R. C. \& Reid, J. S. (1995). Acta Cryst. A51, 887-897.

Dolomanov, O. V., Bourhis, L. J., Gildea, R. J., Howard, J. A. K. \& Puschmann, H. (2009). J. Appl. Cryst. 42, 339-341.

George, A., Brandt, A., Tran, K., Zahari, S. M. S. N. S., KleinMarcuschamer, D., Sun, N., Sathitsuksanoh, N., Shi, J., Stavila, V., Parthasarathi, R., Singh, S., Holmes, B. M., Welton, T., Simmons, B. A. \& Hallett, J. P. (2015). Green Chem. 17, 1728-1734.
Table 2

Experimental details.

\begin{tabular}{|c|c|}
\hline \multicolumn{2}{|l|}{ Crystal data } \\
\hline Chemical formula & $3 \mathrm{C}_{5} \mathrm{H}_{12} \mathrm{~N}^{+} \cdot \mathrm{HSO}_{4}{ }^{-} \cdot \mathrm{SO}_{4}{ }^{2-}$ \\
\hline$M_{\mathrm{r}}$ & 451.60 \\
\hline Crystal system, space group & Monoclinic, $P 2_{1} / c$ \\
\hline Temperature (K) & 100 \\
\hline$a, b, c(\AA)$ & $10.592(4), 17.922(5), 11.161(4)$ \\
\hline$\beta\left({ }^{\circ}\right)$ & $99.25(2)$ \\
\hline$V\left(\AA^{3}\right)$ & $2091.1(12)$ \\
\hline$Z$ & 4 \\
\hline Radiation type & Mo $K \alpha$ \\
\hline$\mu\left(\mathrm{mm}^{-1}\right)$ & 0.30 \\
\hline Crystal size $(\mathrm{mm})$ & $0.20 \times 0.18 \times 0.16$ \\
\hline \multicolumn{2}{|l|}{ Data collection } \\
\hline Diffractometer & Rigaku Oxford Xcalibur Atlas \\
\hline Absorption correction & $\begin{array}{l}\text { Analytical [CrysAlis PRO (Rigaku } \\
\text { Oxford, 2015), based on } \\
\text { expressions of Clark \& Reid } \\
(1995)]\end{array}$ \\
\hline$T_{\min }, T_{\max }$ & $0.994,0.996$ \\
\hline $\begin{array}{l}\text { No. of measured, independent and } \\
\text { observed }[I>2 \sigma(I)] \text { reflections }\end{array}$ & $35669,5411,4291$ \\
\hline$R_{\text {int }}$ & 0.039 \\
\hline$(\sin \theta / \lambda)_{\max }\left(\AA^{-1}\right)$ & 0.691 \\
\hline \multicolumn{2}{|l|}{ Refinement } \\
\hline$R\left[F^{2}>2 \sigma\left(F^{2}\right)\right], w R\left(F^{2}\right), S$ & $0.035,0.083,1.03$ \\
\hline No. of reflections & 5411 \\
\hline No. of parameters & 254 \\
\hline $\mathrm{H}$-atom treatment & $\mathrm{H}$-atom parameters constrained \\
\hline$\Delta \rho_{\max }, \Delta \rho_{\min }\left(\mathrm{e} \AA^{-3}\right)$ & $0.33,-0.42$ \\
\hline
\end{tabular}

Computer programs: CrysAlis PRO (Rigaku Oxford, 2015), SHELXS97 (Sheldrick, 2008), SHELXL2014 (Sheldrick, 2015), DIAMOND (Brandenburg, 1997) and OLEX2 (Dolomanov et al., 2009).

Hoque, M. N. \& Das, G. (2014). Cryst. Growth Des. 14, 2962-2971. Kolev, T., Koleva, B. B., Seidel, R. W., Spiteller, M. \& Sheldrick, W. S. (2009). Cryst. Growth Des. 9, 3348-3352.

Lee, C. \& Harrison, W. T. A. (2003). Acta Cryst. E59, m959-m960.

Mohammadnezhad, G. S., Amini, M. M., Khavasi, H. R. \& Ng, S. W. (2008). Acta Cryst. E64, o1564.

Rigaku Oxford (2015). CrysAlis PRO. Rigaku Corporation, Yarnton, England.

Sheldrick, G. M. (2008). Acta Cryst. A64, 112-122.

Sheldrick, G. M. (2015). Acta Cryst. C71, 3-8.

Smail, E. J. \& Sheldrick, G. M. (1973). Acta Cryst. B29, 2027-2028.

Xu, Y.-M., Gao, S. \& Ng, S. W. (2009). Acta Cryst. E65, o3147. 


\section{supporting information}

Acta Cryst. (2015). E71, 1444-1446 [https://doi.org/10.1107/S2056989015020538]

\section{Crystal structure of tris(piperidinium) hydrogen sulfate sulfate}

\section{Tamara J. Lukianova, Vasyl Kinzhybalo and Adam Pietraszko}

\section{Computing details}

Data collection: CrysAlis PRO (Rigaku Oxford, 2015); cell refinement: CrysAlis PRO (Rigaku Oxford, 2015); data reduction: CrysAlis PRO (Rigaku Oxford, 2015); program(s) used to solve structure: SHELXS97 (Sheldrick, 2008); program(s) used to refine structure: SHELXL2014 (Sheldrick, 2015); molecular graphics: DIAMOND (Brandenburg, 1997); software used to prepare material for publication: OLEX2 (Dolomanov et al., 2009).

Tris(piperidinium) hydrogen sulfate sulfate

Crystal data

$$
\begin{aligned}
& 3 \mathrm{C}_{5} \mathrm{H}_{12} \mathrm{~N}^{+} \cdot \mathrm{HSO}_{4} \cdot \mathrm{SO}_{4}{ }^{2-} \\
& M_{r}=451.60 \\
& \text { Monoclinic, } P 2_{1} / c \\
& a=10.592(4) \AA \\
& b=17.922(5) \AA \\
& c=11.161(4) \AA \\
& \beta=99.25(2)^{\circ} \\
& V=2091.1(12) \AA^{3} \\
& Z=4
\end{aligned}
$$

Data collection

Rigaku Oxford Xcalibur Atlas diffractometer

Radiation source: fine-focus sealed X-ray tube, Enhance (Mo) X-ray source

Graphite monochromator

Detector resolution: 10.6249 pixels $\mathrm{mm}^{-1}$

$\omega$ scans

Absorption correction: analytical

[CrysAlis PRO (Rigaku Oxford, 2015), based on expressions of Clark \& Reid (1995)]

\section{Refinement}

Refinement on $F^{2}$

Least-squares matrix: full

$R\left[F^{2}>2 \sigma\left(F^{2}\right)\right]=0.035$

$w R\left(F^{2}\right)=0.083$

$S=1.03$

5411 reflections 254 parameters 0 restraints
$F(000)=976$

$D_{\mathrm{x}}=1.434 \mathrm{Mg} \mathrm{m}^{-3}$

Mo $K \alpha$ radiation, $\lambda=0.71073 \AA$

Cell parameters from 12552 reflections

$\theta=2.2-29.4^{\circ}$

$\mu=0.30 \mathrm{~mm}^{-1}$

$T=100 \mathrm{~K}$

Block, colourless

$0.20 \times 0.18 \times 0.16 \mathrm{~mm}$

$T_{\min }=0.994, T_{\max }=0.996$

35669 measured reflections

5411 independent reflections

4291 reflections with $I>2 \sigma(I)$

$R_{\text {int }}=0.039$

$\theta_{\max }=29.4^{\circ}, \theta_{\min }=2.7^{\circ}$

$h=-14 \rightarrow 14$

$k=-23 \rightarrow 24$

$l=-15 \rightarrow 15$

Hydrogen site location: inferred from neighbouring sites

$\mathrm{H}$-atom parameters constrained

$w=1 /\left[\sigma^{2}\left(F_{\mathrm{o}}^{2}\right)+(0.0343 P)^{2}+1.1863 P\right]$

where $P=\left(F_{\mathrm{o}}^{2}+2 F_{\mathrm{c}}^{2}\right) / 3$

$(\Delta / \sigma)_{\max }=0.001$

$\Delta \rho_{\max }=0.33$ e $\AA^{-3}$

$\Delta \rho_{\text {min }}=-0.42$ e $\AA^{-3}$ 


\section{Special details}

Geometry. All e.s.d.'s (except the e.s.d. in the dihedral angle between two 1.s. planes) are estimated using the full covariance matrix. The cell e.s.d.'s are taken into account individually in the estimation of e.s.d.'s in distances, angles and torsion angles; correlations between e.s.d.'s in cell parameters are only used when they are defined by crystal symmetry. An approximate (isotropic) treatment of cell e.s.d.'s is used for estimating e.s.d.'s involving l.s. planes.

Fractional atomic coordinates and isotropic or equivalent isotropic displacement parameters $\left(\hat{A}^{2}\right)$

\begin{tabular}{|c|c|c|c|c|}
\hline & $x$ & $y$ & $z$ & $U_{\text {iso }} * / U_{\text {eq }}$ \\
\hline S1 & $0.66217(3)$ & $0.57717(2)$ & $0.56541(3)$ & $0.01118(9)$ \\
\hline S2 & $0.39851(3)$ & $0.75550(2)$ & $0.40181(3)$ & $0.01134(9)$ \\
\hline $\mathrm{O} 12$ & $0.57890(10)$ & $0.58513(6)$ & $0.65614(9)$ & $0.0155(2)$ \\
\hline $\mathrm{O} 21$ & $0.39138(10)$ & $0.67389(6)$ & $0.42549(10)$ & $0.0183(2)$ \\
\hline O14 & $0.57685(10)$ & $0.58114(6)$ & $0.43754(10)$ & $0.0175(2)$ \\
\hline H14 & 0.5194 & 0.6134 & 0.4385 & $0.026^{*}$ \\
\hline O11 & $0.72505(10)$ & $0.50505(6)$ & $0.56684(10)$ & $0.0174(2)$ \\
\hline $\mathrm{O} 13$ & $0.75266(10)$ & $0.63911(6)$ & $0.57133(10)$ & $0.0180(2)$ \\
\hline $\mathrm{O} 22$ & $0.50129(11)$ & $0.79014(7)$ & $0.48587(10)$ & $0.0222(3)$ \\
\hline $\mathrm{C} 25$ & $0.19519(15)$ & $0.50258(8)$ & $0.72404(14)$ & $0.0154(3)$ \\
\hline $\mathrm{H} 25 \mathrm{~A}$ & 0.2000 & 0.4735 & 0.8001 & $0.018^{*}$ \\
\hline $\mathrm{H} 25 \mathrm{~B}$ & 0.1955 & 0.4669 & 0.6563 & $0.018^{*}$ \\
\hline $\mathrm{N} 21$ & $0.30210(12)$ & $0.60129(7)$ & $0.62212(11)$ & $0.0127(3)$ \\
\hline $\mathrm{H} 21 \mathrm{~A}$ & 0.3029 & 0.5718 & 0.5559 & $0.015^{*}$ \\
\hline $\mathrm{H} 21 \mathrm{~B}$ & 0.3716 & 0.6318 & 0.6293 & $0.015^{*}$ \\
\hline $\mathrm{O} 23$ & $0.27443(10)$ & $0.78993(6)$ & $0.41375(9)$ & $0.0142(2)$ \\
\hline $\mathrm{O} 24$ & $0.42147(10)$ & $0.76415(6)$ & $0.27366(9)$ & $0.0135(2)$ \\
\hline N11 & $0.32098(12)$ & $0.63977(7)$ & $0.14102(11)$ & $0.0131(3)$ \\
\hline H11A & 0.3570 & 0.6768 & 0.1909 & $0.016^{*}$ \\
\hline H11B & 0.3156 & 0.6560 & 0.0631 & $0.016^{*}$ \\
\hline N31 & $0.67984(12)$ & $0.73600(7)$ & $0.74774(12)$ & $0.0159(3)$ \\
\hline $\mathrm{H} 31 \mathrm{~A}$ & 0.6901 & 0.7023 & 0.6890 & $0.019^{*}$ \\
\hline H31B & 0.5947 & 0.7394 & 0.7510 & $0.019^{*}$ \\
\hline C14 & $0.21075(15)$ & $0.49250(8)$ & $0.09492(14)$ & $0.0164(3)$ \\
\hline $\mathrm{H} 14 \mathrm{~A}$ & 0.2125 & 0.4710 & 0.1768 & $0.020^{*}$ \\
\hline H14B & 0.1725 & 0.4551 & 0.0345 & $0.020^{*}$ \\
\hline $\mathrm{C} 26$ & $0.31146(14)$ & $0.55327(8)$ & $0.73257(13)$ & $0.0138(3)$ \\
\hline $\mathrm{H} 26 \mathrm{~A}$ & 0.3169 & 0.5851 & 0.8057 & $0.017^{*}$ \\
\hline H26B & 0.3901 & 0.5226 & 0.7403 & $0.017^{*}$ \\
\hline $\mathrm{C} 23$ & $0.06623(15)$ & $0.59747(9)$ & $0.59190(15)$ & $0.0176(3)$ \\
\hline $\mathrm{H} 23 \mathrm{~A}$ & 0.0622 & 0.5662 & 0.5183 & $0.021^{*}$ \\
\hline H23B & -0.0121 & 0.6285 & 0.5828 & $0.021 *$ \\
\hline C16 & $0.40461(14)$ & $0.57238(8)$ & $0.15854(14)$ & $0.0152(3)$ \\
\hline H16A & 0.4139 & 0.5557 & 0.2441 & $0.018^{*}$ \\
\hline H16B & 0.4907 & 0.5850 & 0.1406 & $0.018^{*}$ \\
\hline $\mathrm{C} 22$ & $0.18310(14)$ & $0.64782(8)$ & $0.60349(14)$ & $0.0155(3)$ \\
\hline $\mathrm{H} 22 \mathrm{~A}$ & 0.1799 & 0.6782 & 0.5290 & $0.019^{*}$ \\
\hline H22B & 0.1837 & 0.6821 & 0.6731 & $0.019^{*}$ \\
\hline $\mathrm{C} 24$ & $0.07120(15)$ & $0.54724(9)$ & $0.70327(15)$ & $0.0189(3)$ \\
\hline
\end{tabular}




$\begin{array}{lllll}\text { H24A } & 0.0655 & 0.5782 & 0.7757 & 0.023^{*} \\ \text { H24B } & -0.0027 & 0.5127 & 0.6912 & 0.023^{*} \\ \text { C12 } & 0.18991(14) & 0.62418(9) & 0.16711(14) & 0.0160(3) \\ \text { H12A } & 0.1374 & 0.6701 & 0.1552 & 0.019^{*} \\ \text { H12B } & 0.1946 & 0.6080 & 0.2525 & 0.019^{*} \\ \text { C33 } & 0.86970(15) & 0.80450(9) & 0.70869(15) & 0.0180(3) \\ \text { H33A } & 0.8828 & 0.7698 & 0.6428 & 0.022^{*} \\ \text { H33B } & 0.9023 & 0.8541 & 0.6895 & 0.022^{*} \\ \text { C35 } & 0.89016(16) & 0.70228(10) & 0.86207(16) & 0.0217(4) \\ \text { H35A } & 0.9040 & 0.6641 & 0.8014 & 0.026^{*} \\ \text { H35B } & 0.9357 & 0.6860 & 0.9422 & 0.026^{*} \\ \text { C15 } & 0.34720(15) & 0.50991(8) & 0.07536(14) & 0.0161(3) \\ \text { H15A } & 0.4007 & 0.4646 & 0.0915 & 0.019^{*} \\ \text { H15B } & 0.3466 & 0.5247 & -0.0102 & 0.019^{*} \\ \text { C13 } & 0.12870(15) & 0.56320(9) & 0.08232(15) & 0.0169(3) \\ \text { H13A } & 0.1182 & 0.5812 & -0.0026 & 0.020^{*} \\ \text { H13B } & 0.0428 & 0.5515 & 0.1015 & 0.020^{*} \\ \text { C32 } & 0.72742(14) & 0.81012(8) & 0.71454(15) & 0.0167(3) \\ \text { H32A } & 0.7129 & 0.8477 & 0.7758 & 0.020^{*} \\ \text { H32B } & 0.6804 & 0.8260 & 0.6348 & 0.020^{*} \\ \text { C36 } & 0.74807(16) & 0.70877(9) & 0.86681(16) & 0.0205(3) \\ \text { H36A } & 0.7138 & 0.6595 & 0.8854 & 0.025^{*} \\ \text { H36B } & 0.7343 & 0.7440 & 0.9318 & 0.025^{*} \\ \text { C34 } & 0.94462(15) & 0.77687(10) & 0.82831(15) & 0.0207(3) \\ \text { H34A } & 0.9390 & 0.8139 & 0.8930 & 0.025^{*} \\ \text { H34B } & 1.0358 & 0.7708 & 0.8205 & 0.025^{*} \\ & & & & \end{array}$

Atomic displacement parameters $\left(\AA^{2}\right)$

\begin{tabular}{lllllll}
\hline & $U^{11}$ & $U^{22}$ & $U^{33}$ & $U^{12}$ & $U^{13}$ & $U^{23}$ \\
\hline $\mathrm{S} 1$ & $0.01134(17)$ & $0.01084(17)$ & $0.01124(17)$ & $0.00075(13)$ & $0.00142(13)$ & $-0.00154(13)$ \\
$\mathrm{S} 2$ & $0.01054(17)$ & $0.01264(17)$ & $0.01101(17)$ & $0.00085(13)$ & $0.00228(13)$ & $-0.00016(13)$ \\
$\mathrm{O} 12$ & $0.0153(5)$ & $0.0173(5)$ & $0.0147(5)$ & $0.0002(4)$ & $0.0049(4)$ & $-0.0014(4)$ \\
$\mathrm{O} 21$ & $0.0207(6)$ & $0.0144(5)$ & $0.0214(6)$ & $0.0051(4)$ & $0.0077(5)$ & $0.0061(4)$ \\
$\mathrm{O} 14$ & $0.0175(6)$ & $0.0209(6)$ & $0.0127(5)$ & $0.0071(4)$ & $-0.0014(4)$ & $-0.0035(4)$ \\
$\mathrm{O} 11$ & $0.0208(6)$ & $0.0132(5)$ & $0.0174(6)$ & $0.0054(4)$ & $0.0013(5)$ & $-0.0018(4)$ \\
$\mathrm{O} 13$ & $0.0147(5)$ & $0.0157(5)$ & $0.0246(6)$ & $-0.0029(4)$ & $0.0063(5)$ & $-0.0038(4)$ \\
$\mathrm{O} 22$ & $0.0144(5)$ & $0.0339(7)$ & $0.0180(6)$ & $-0.0035(5)$ & $0.0013(5)$ & $-0.0088(5)$ \\
$\mathrm{C} 25$ & $0.0189(8)$ & $0.0125(7)$ & $0.0154(7)$ & $-0.0006(6)$ & $0.0046(6)$ & $0.0019(6)$ \\
$\mathrm{N} 21$ & $0.0109(6)$ & $0.0133(6)$ & $0.0140(6)$ & $-0.0009(5)$ & $0.0023(5)$ & $0.0015(5)$ \\
$\mathrm{O} 23$ & $0.0130(5)$ & $0.0152(5)$ & $0.0152(5)$ & $0.0029(4)$ & $0.0043(4)$ & $0.0004(4)$ \\
$\mathrm{O} 24$ & $0.0147(5)$ & $0.0141(5)$ & $0.0124(5)$ & $0.0001(4)$ & $0.0041(4)$ & $0.0008(4)$ \\
$\mathrm{N} 11$ & $0.0151(6)$ & $0.0129(6)$ & $0.0114(6)$ & $-0.0017(5)$ & $0.0023(5)$ & $0.0004(5)$ \\
$\mathrm{N} 31$ & $0.0110(6)$ & $0.0143(6)$ & $0.0231(7)$ & $-0.0014(5)$ & $0.0046(5)$ & $-0.0073(5)$ \\
$\mathrm{C} 14$ & $0.0169(7)$ & $0.0141(7)$ & $0.0168(8)$ & $-0.0024(6)$ & $-0.0017(6)$ & $-0.0003(6)$ \\
$\mathrm{C} 26$ & $0.0139(7)$ & $0.0143(7)$ & $0.0127(7)$ & $0.0006(6)$ & $0.0008(6)$ & $0.0028(6)$ \\
$\mathrm{C} 23$ & $0.0122(7)$ & $0.0232(8)$ & $0.0177(8)$ & $0.0023(6)$ & $0.0027(6)$ & $0.0040(6)$ \\
$\mathrm{C} 16$ & $0.0128(7)$ & $0.0157(7)$ & $0.0163(7)$ & $0.0006(6)$ & $0.0000(6)$ & $0.0016(6)$
\end{tabular}




\begin{tabular}{lllllll} 
C22 & $0.0159(7)$ & $0.0140(7)$ & $0.0168(7)$ & $0.0040(6)$ & $0.0034(6)$ & $0.0034(6)$ \\
C24 & $0.0151(7)$ & $0.0226(8)$ & $0.0200(8)$ & $-0.0014(6)$ & $0.0058(6)$ & $0.0039(6)$ \\
C12 & $0.0138(7)$ & $0.0170(7)$ & $0.0177(8)$ & $-0.0006(6)$ & $0.0042(6)$ & $-0.0001(6)$ \\
C33 & $0.0148(7)$ & $0.0159(7)$ & $0.0236(8)$ & $-0.0010(6)$ & $0.0044(7)$ & $0.0003(6)$ \\
C35 & $0.0195(8)$ & $0.0271(9)$ & $0.0196(8)$ & $0.0088(7)$ & $0.0061(7)$ & $0.0044(7)$ \\
C15 & $0.0168(8)$ & $0.0148(7)$ & $0.0162(7)$ & $0.0008(6)$ & $0.0010(6)$ & $-0.0002(6)$ \\
C13 & $0.0132(7)$ & $0.0173(7)$ & $0.0194(8)$ & $-0.0015(6)$ & $0.0001(6)$ & $0.0006(6)$ \\
C32 & $0.0140(7)$ & $0.0147(7)$ & $0.0208(8)$ & $0.0008(6)$ & $0.0012(6)$ & $-0.0004(6)$ \\
C36 & $0.0210(8)$ & $0.0185(8)$ & $0.0242(9)$ & $0.0035(6)$ & $0.0106(7)$ & $0.0043(6)$ \\
C34 & $0.0124(7)$ & $0.0273(9)$ & $0.0217(8)$ & $-0.0013(6)$ & $0.0009(6)$ & $-0.0050(7)$ \\
\hline
\end{tabular}

Geometric parameters $\left(\AA,{ }^{\circ}\right)$

\begin{tabular}{|c|c|c|c|}
\hline $\mathrm{S} 1-\mathrm{O} 12$ & $1.4529(12)$ & $\mathrm{C} 23-\mathrm{H} 23 \mathrm{~B}$ & 0.9900 \\
\hline $\mathrm{S} 1-\mathrm{O} 14$ & $1.5633(12)$ & $\mathrm{C} 23-\mathrm{C} 22$ & $1.520(2)$ \\
\hline $\mathrm{S} 1-\mathrm{O} 11$ & $1.4530(11)$ & $\mathrm{C} 23-\mathrm{C} 24$ & $1.529(2)$ \\
\hline $\mathrm{S} 1-\mathrm{O} 13$ & $1.4612(11)$ & $\mathrm{C} 16-\mathrm{H} 16 \mathrm{~A}$ & 0.9900 \\
\hline $\mathrm{S} 2-\mathrm{O} 21$ & $1.4904(12)$ & C16-H16B & 0.9900 \\
\hline $\mathrm{S} 2-\mathrm{O} 22$ & $1.4569(12)$ & $\mathrm{C} 16-\mathrm{C} 15$ & $1.518(2)$ \\
\hline $\mathrm{S} 2-\mathrm{O} 23$ & $1.4773(11)$ & $\mathrm{C} 22-\mathrm{H} 22 \mathrm{~A}$ & 0.9900 \\
\hline $\mathrm{S} 2-\mathrm{O} 24$ & $1.4969(12)$ & $\mathrm{C} 22-\mathrm{H} 22 \mathrm{~B}$ & 0.9900 \\
\hline $\mathrm{O} 14-\mathrm{H} 14$ & 0.8400 & $\mathrm{C} 24-\mathrm{H} 24 \mathrm{~A}$ & 0.9900 \\
\hline $\mathrm{C} 25-\mathrm{H} 25 \mathrm{~A}$ & 0.9900 & $\mathrm{C} 24-\mathrm{H} 24 \mathrm{~B}$ & 0.9900 \\
\hline $\mathrm{C} 25-\mathrm{H} 25 \mathrm{~B}$ & 0.9900 & $\mathrm{C} 12-\mathrm{H} 12 \mathrm{~A}$ & 0.9900 \\
\hline $\mathrm{C} 25-\mathrm{C} 26$ & $1.521(2)$ & $\mathrm{C} 12-\mathrm{H} 12 \mathrm{~B}$ & 0.9900 \\
\hline $\mathrm{C} 25-\mathrm{C} 24$ & $1.523(2)$ & $\mathrm{C} 12-\mathrm{C} 13$ & $1.521(2)$ \\
\hline $\mathrm{N} 21-\mathrm{H} 21 \mathrm{~A}$ & 0.9100 & $\mathrm{C} 33-\mathrm{H} 33 \mathrm{~A}$ & 0.9900 \\
\hline $\mathrm{N} 21-\mathrm{H} 21 \mathrm{~B}$ & 0.9100 & $\mathrm{C} 33-\mathrm{H} 33 \mathrm{~B}$ & 0.9900 \\
\hline $\mathrm{N} 21-\mathrm{C} 26$ & $1.4936(19)$ & $\mathrm{C} 33-\mathrm{C} 32$ & $1.522(2)$ \\
\hline $\mathrm{N} 21-\mathrm{C} 22$ & $1.4978(19)$ & $\mathrm{C} 33-\mathrm{C} 34$ & $1.522(2)$ \\
\hline $\mathrm{N} 11-\mathrm{H} 11 \mathrm{~A}$ & 0.9100 & $\mathrm{C} 35-\mathrm{H} 35 \mathrm{~A}$ & 0.9900 \\
\hline N11-H11B & 0.9100 & $\mathrm{C} 35-\mathrm{H} 35 \mathrm{~B}$ & 0.9900 \\
\hline N11-C16 & $1.4920(19)$ & $\mathrm{C} 35-\mathrm{C} 36$ & $1.519(2)$ \\
\hline $\mathrm{N} 11-\mathrm{C} 12$ & $1.4900(19)$ & $\mathrm{C} 35-\mathrm{C} 34$ & $1.527(2)$ \\
\hline $\mathrm{N} 31-\mathrm{H} 31 \mathrm{~A}$ & 0.9100 & $\mathrm{C} 15-\mathrm{H} 15 \mathrm{~A}$ & 0.9900 \\
\hline N31-H31B & 0.9100 & C15-H15B & 0.9900 \\
\hline $\mathrm{N} 31-\mathrm{C} 32$ & $1.489(2)$ & $\mathrm{C} 13-\mathrm{H} 13 \mathrm{~A}$ & 0.9900 \\
\hline $\mathrm{N} 31-\mathrm{C} 36$ & $1.489(2)$ & $\mathrm{C} 13-\mathrm{H} 13 \mathrm{~B}$ & 0.9900 \\
\hline $\mathrm{C} 14-\mathrm{H} 14 \mathrm{~A}$ & 0.9900 & $\mathrm{C} 32-\mathrm{H} 32 \mathrm{~A}$ & 0.9900 \\
\hline C14-H14B & 0.9900 & $\mathrm{C} 32-\mathrm{H} 32 \mathrm{~B}$ & 0.9900 \\
\hline $\mathrm{C} 14-\mathrm{C} 15$ & $1.528(2)$ & $\mathrm{C} 36-\mathrm{H} 36 \mathrm{~A}$ & 0.9900 \\
\hline $\mathrm{C} 14-\mathrm{C} 13$ & $1.530(2)$ & $\mathrm{C} 36-\mathrm{H} 36 \mathrm{~B}$ & 0.9900 \\
\hline $\mathrm{C} 26-\mathrm{H} 26 \mathrm{~A}$ & 0.9900 & $\mathrm{C} 34-\mathrm{H} 34 \mathrm{~A}$ & 0.9900 \\
\hline $\mathrm{C} 26-\mathrm{H} 26 \mathrm{~B}$ & 0.9900 & C34-H34B & 0.9900 \\
\hline $\mathrm{C} 23-\mathrm{H} 23 \mathrm{~A}$ & 0.9900 & & \\
\hline $\mathrm{O} 12-\mathrm{S} 1-\mathrm{O} 14$ & $107.77(7)$ & $\mathrm{N} 21-\mathrm{C} 22-\mathrm{C} 23$ & $109.68(12)$ \\
\hline $\mathrm{O} 12-\mathrm{S} 1-\mathrm{O} 11$ & $114.10(7)$ & $\mathrm{N} 21-\mathrm{C} 22-\mathrm{H} 22 \mathrm{~A}$ & 109.7 \\
\hline
\end{tabular}




\begin{tabular}{|c|c|}
\hline $\mathrm{O} 12-\mathrm{S} 1-\mathrm{O} 13$ & $111.17(7)$ \\
\hline $\mathrm{O} 11-\mathrm{S} 1-\mathrm{O} 14$ & $104.31(6)$ \\
\hline $\mathrm{O} 11-\mathrm{S} 1-\mathrm{O} 13$ & $112.28(7)$ \\
\hline $\mathrm{O} 13-\mathrm{S} 1-\mathrm{O} 14$ & $106.58(7)$ \\
\hline $\mathrm{O} 21-\mathrm{S} 2-\mathrm{O} 24$ & $106.96(6)$ \\
\hline $\mathrm{O} 22-\mathrm{S} 2-\mathrm{O} 21$ & $110.98(7)$ \\
\hline $\mathrm{O} 22-\mathrm{S} 2-\mathrm{O} 23$ & $110.32(7)$ \\
\hline $\mathrm{O} 22-\mathrm{S} 2-\mathrm{O} 24$ & $110.60(7)$ \\
\hline $\mathrm{O} 23-\mathrm{S} 2-\mathrm{O} 21$ & $108.83(6)$ \\
\hline $\mathrm{O} 23-\mathrm{S} 2-\mathrm{O} 24$ & $109.05(6)$ \\
\hline $\mathrm{S} 1-\mathrm{O} 14-\mathrm{H} 14$ & 109.5 \\
\hline $\mathrm{H} 25 \mathrm{~A}-\mathrm{C} 25-\mathrm{H} 25 \mathrm{~B}$ & 108.0 \\
\hline $\mathrm{C} 26-\mathrm{C} 25-\mathrm{H} 25 \mathrm{~A}$ & 109.3 \\
\hline $\mathrm{C} 26-\mathrm{C} 25-\mathrm{H} 25 \mathrm{~B}$ & 109.3 \\
\hline $\mathrm{C} 26-\mathrm{C} 25-\mathrm{C} 24$ & $111.42(13)$ \\
\hline $\mathrm{C} 24-\mathrm{C} 25-\mathrm{H} 25 \mathrm{~A}$ & 109.3 \\
\hline $\mathrm{C} 24-\mathrm{C} 25-\mathrm{H} 25 \mathrm{~B}$ & 109.3 \\
\hline $\mathrm{H} 21 \mathrm{~A}-\mathrm{N} 21-\mathrm{H} 21 \mathrm{~B}$ & 107.9 \\
\hline $\mathrm{C} 26-\mathrm{N} 21-\mathrm{H} 21 \mathrm{~A}$ & 109.2 \\
\hline $\mathrm{C} 26-\mathrm{N} 21-\mathrm{H} 21 \mathrm{~B}$ & 109.2 \\
\hline $\mathrm{C} 26-\mathrm{N} 21-\mathrm{C} 22$ & $112.22(11)$ \\
\hline $\mathrm{C} 22-\mathrm{N} 21-\mathrm{H} 21 \mathrm{~A}$ & 109.2 \\
\hline $\mathrm{C} 22-\mathrm{N} 21-\mathrm{H} 21 \mathrm{~B}$ & 109.2 \\
\hline $\mathrm{H} 11 \mathrm{~A}-\mathrm{N} 11-\mathrm{H} 11 \mathrm{~B}$ & 107.9 \\
\hline $\mathrm{C} 16-\mathrm{N} 11-\mathrm{H} 11 \mathrm{~A}$ & 109.2 \\
\hline $\mathrm{C} 16-\mathrm{N} 11-\mathrm{H} 11 \mathrm{~B}$ & 109.2 \\
\hline $\mathrm{C} 12-\mathrm{N} 11-\mathrm{H} 11 \mathrm{~A}$ & 109.2 \\
\hline $\mathrm{C} 12-\mathrm{N} 11-\mathrm{H} 11 \mathrm{~B}$ & 109.2 \\
\hline $\mathrm{C} 12-\mathrm{N} 11-\mathrm{C} 16$ & $112.01(11)$ \\
\hline $\mathrm{H} 31 \mathrm{~A}-\mathrm{N} 31-\mathrm{H} 31 \mathrm{~B}$ & 107.9 \\
\hline $\mathrm{C} 32-\mathrm{N} 31-\mathrm{H} 31 \mathrm{~A}$ & 109.1 \\
\hline $\mathrm{C} 32-\mathrm{N} 31-\mathrm{H} 31 \mathrm{~B}$ & 109.1 \\
\hline $\mathrm{C} 32-\mathrm{N} 31-\mathrm{C} 36$ & $112.30(12)$ \\
\hline $\mathrm{C} 36-\mathrm{N} 31-\mathrm{H} 31 \mathrm{~A}$ & 109.1 \\
\hline $\mathrm{C} 36-\mathrm{N} 31-\mathrm{H} 31 \mathrm{~B}$ & 109.1 \\
\hline $\mathrm{H} 14 \mathrm{~A}-\mathrm{C} 14-\mathrm{H} 14 \mathrm{~B}$ & 108.1 \\
\hline $\mathrm{C} 15-\mathrm{C} 14-\mathrm{H} 14 \mathrm{~A}$ & 109.5 \\
\hline $\mathrm{C} 15-\mathrm{C} 14-\mathrm{H} 14 \mathrm{~B}$ & 109.5 \\
\hline $\mathrm{C} 15-\mathrm{C} 14-\mathrm{C} 13$ & $110.74(12)$ \\
\hline $\mathrm{C} 13-\mathrm{C} 14-\mathrm{H} 14 \mathrm{~A}$ & 109.5 \\
\hline $\mathrm{C} 13-\mathrm{C} 14-\mathrm{H} 14 \mathrm{~B}$ & 109.5 \\
\hline $\mathrm{C} 25-\mathrm{C} 26-\mathrm{H} 26 \mathrm{~A}$ & 109.6 \\
\hline $\mathrm{C} 25-\mathrm{C} 26-\mathrm{H} 26 \mathrm{~B}$ & 109.6 \\
\hline $\mathrm{N} 21-\mathrm{C} 26-\mathrm{C} 25$ & $110.29(12)$ \\
\hline $\mathrm{N} 21-\mathrm{C} 26-\mathrm{H} 26 \mathrm{~A}$ & 109.6 \\
\hline $\mathrm{N} 21-\mathrm{C} 26-\mathrm{H} 26 \mathrm{~B}$ & 109.6 \\
\hline $\mathrm{H} 26 \mathrm{~A}-\mathrm{C} 26-\mathrm{H} 26 \mathrm{~B}$ & 108.1 \\
\hline & \\
\hline
\end{tabular}

\begin{tabular}{|c|c|}
\hline $\mathrm{N} 21-\mathrm{C} 22-\mathrm{H} 22 \mathrm{~B}$ & 109.7 \\
\hline $\mathrm{C} 23-\mathrm{C} 22-\mathrm{H} 22 \mathrm{~A}$ & 109.7 \\
\hline $\mathrm{C} 23-\mathrm{C} 22-\mathrm{H} 22 \mathrm{~B}$ & 109.7 \\
\hline $\mathrm{H} 22 \mathrm{~A}-\mathrm{C} 22-\mathrm{H} 22 \mathrm{~B}$ & 108.2 \\
\hline $\mathrm{C} 25-\mathrm{C} 24-\mathrm{C} 23$ & $110.44(13)$ \\
\hline $\mathrm{C} 25-\mathrm{C} 24-\mathrm{H} 24 \mathrm{~A}$ & 109.6 \\
\hline $\mathrm{C} 25-\mathrm{C} 24-\mathrm{H} 24 \mathrm{~B}$ & 109.6 \\
\hline $\mathrm{C} 23-\mathrm{C} 24-\mathrm{H} 24 \mathrm{~A}$ & 109.6 \\
\hline $\mathrm{C} 23-\mathrm{C} 24-\mathrm{H} 24 \mathrm{~B}$ & 109.6 \\
\hline $\mathrm{H} 24 \mathrm{~A}-\mathrm{C} 24-\mathrm{H} 24 \mathrm{~B}$ & 108.1 \\
\hline $\mathrm{N} 11-\mathrm{C} 12-\mathrm{H} 12 \mathrm{~A}$ & 109.8 \\
\hline $\mathrm{N} 11-\mathrm{C} 12-\mathrm{H} 12 \mathrm{~B}$ & 109.8 \\
\hline $\mathrm{N} 11-\mathrm{C} 12-\mathrm{C} 13$ & $109.30(12)$ \\
\hline $\mathrm{H} 12 \mathrm{~A}-\mathrm{C} 12-\mathrm{H} 12 \mathrm{~B}$ & 108.3 \\
\hline $\mathrm{C} 13-\mathrm{C} 12-\mathrm{H} 12 \mathrm{~A}$ & 109.8 \\
\hline $\mathrm{C} 13-\mathrm{C} 12-\mathrm{H} 12 \mathrm{~B}$ & 109.8 \\
\hline $\mathrm{H} 33 \mathrm{~A}-\mathrm{C} 33-\mathrm{H} 33 \mathrm{~B}$ & 108.0 \\
\hline $\mathrm{C} 32-\mathrm{C} 33-\mathrm{H} 33 \mathrm{~A}$ & 109.4 \\
\hline C $32-\mathrm{C} 33-\mathrm{H} 33 \mathrm{~B}$ & 109.4 \\
\hline $\mathrm{C} 34-\mathrm{C} 33-\mathrm{H} 33 \mathrm{~A}$ & 109.4 \\
\hline $\mathrm{C} 34-\mathrm{C} 33-\mathrm{H} 33 \mathrm{~B}$ & 109.4 \\
\hline $\mathrm{C} 34-\mathrm{C} 33-\mathrm{C} 32$ & $111.35(14)$ \\
\hline $\mathrm{H} 35 \mathrm{~A}-\mathrm{C} 35-\mathrm{H} 35 \mathrm{~B}$ & 108.0 \\
\hline $\mathrm{C} 36-\mathrm{C} 35-\mathrm{H} 35 \mathrm{~A}$ & 109.5 \\
\hline $\mathrm{C} 36-\mathrm{C} 35-\mathrm{H} 35 \mathrm{~B}$ & 109.5 \\
\hline $\mathrm{C} 36-\mathrm{C} 35-\mathrm{C} 34$ & $110.94(13)$ \\
\hline $\mathrm{C} 34-\mathrm{C} 35-\mathrm{H} 35 \mathrm{~A}$ & 109.5 \\
\hline $\mathrm{C} 34-\mathrm{C} 35-\mathrm{H} 35 \mathrm{~B}$ & 109.5 \\
\hline $\mathrm{C} 14-\mathrm{C} 15-\mathrm{H} 15 \mathrm{~A}$ & 109.4 \\
\hline $\mathrm{C} 14-\mathrm{C} 15-\mathrm{H} 15 \mathrm{~B}$ & 109.4 \\
\hline $\mathrm{C} 16-\mathrm{C} 15-\mathrm{C} 14$ & $111.00(13)$ \\
\hline $\mathrm{C} 16-\mathrm{C} 15-\mathrm{H} 15 \mathrm{~A}$ & 109.4 \\
\hline $\mathrm{C} 16-\mathrm{C} 15-\mathrm{H} 15 \mathrm{~B}$ & 109.4 \\
\hline $\mathrm{H} 15 \mathrm{~A}-\mathrm{C} 15-\mathrm{H} 15 \mathrm{~B}$ & 108.0 \\
\hline $\mathrm{C} 14-\mathrm{C} 13-\mathrm{H} 13 \mathrm{~A}$ & 109.4 \\
\hline $\mathrm{C} 14-\mathrm{C} 13-\mathrm{H} 13 \mathrm{~B}$ & 109.4 \\
\hline $\mathrm{C} 12-\mathrm{C} 13-\mathrm{C} 14$ & $111.03(13)$ \\
\hline $\mathrm{C} 12-\mathrm{C} 13-\mathrm{H} 13 \mathrm{~A}$ & 109.4 \\
\hline $\mathrm{C} 12-\mathrm{C} 13-\mathrm{H} 13 \mathrm{~B}$ & 109.4 \\
\hline $\mathrm{H} 13 \mathrm{~A}-\mathrm{C} 13-\mathrm{H} 13 \mathrm{~B}$ & 108.0 \\
\hline $\mathrm{N} 31-\mathrm{C} 32-\mathrm{C} 33$ & $109.20(12)$ \\
\hline $\mathrm{N} 31-\mathrm{C} 32-\mathrm{H} 32 \mathrm{~A}$ & 109.8 \\
\hline $\mathrm{N} 31-\mathrm{C} 32-\mathrm{H} 32 \mathrm{~B}$ & 109.8 \\
\hline $\mathrm{C} 33-\mathrm{C} 32-\mathrm{H} 32 \mathrm{~A}$ & 109.8 \\
\hline $\mathrm{C} 33-\mathrm{C} 32-\mathrm{H} 32 \mathrm{~B}$ & 109.8 \\
\hline $\mathrm{H} 32 \mathrm{~A}-\mathrm{C} 32-\mathrm{H} 32 \mathrm{~B}$ & 108.3 \\
\hline $\mathrm{N} 31-\mathrm{C} 36-\mathrm{C} 35$ & $109.60(13)$ \\
\hline $\mathrm{N} 31-\mathrm{C} 36-\mathrm{H} 36 \mathrm{~A}$ & 109.8 \\
\hline
\end{tabular}




$\mathrm{C} 22-\mathrm{C} 23-\mathrm{H} 23 \mathrm{~A}$
$\mathrm{C} 22-\mathrm{C} 23-\mathrm{H} 23 \mathrm{~B}$
$\mathrm{C} 22-\mathrm{C} 23-\mathrm{C} 24$
$\mathrm{C} 24-\mathrm{C} 23-\mathrm{H} 23 \mathrm{~A}$
$\mathrm{C} 24-\mathrm{C} 23-\mathrm{H} 23 \mathrm{~B}$
$\mathrm{~N} 11-\mathrm{C} 16-\mathrm{H} 16 \mathrm{~A}$
$\mathrm{~N} 11-\mathrm{C} 16-\mathrm{H} 16 \mathrm{~B}$
$\mathrm{~N} 11-\mathrm{C} 16-\mathrm{C} 15$
$\mathrm{H} 16 \mathrm{~A}-\mathrm{C} 16-\mathrm{H} 16 \mathrm{~B}$
$\mathrm{C} 15-\mathrm{C} 16-\mathrm{H} 16 \mathrm{~A}$
$\mathrm{C} 15-\mathrm{C} 16-\mathrm{H} 16 \mathrm{~B}$
$\mathrm{~N} 11-\mathrm{C} 16-\mathrm{C} 15-\mathrm{C} 14$
$\mathrm{~N} 11-\mathrm{C} 12-\mathrm{C} 13-\mathrm{C} 14$
$\mathrm{C} 26-\mathrm{C} 25-\mathrm{C} 24-\mathrm{C} 23$
$\mathrm{C} 26-\mathrm{N} 21-\mathrm{C} 22-\mathrm{C} 23$
$\mathrm{C} 16-\mathrm{N} 11-\mathrm{C} 12-\mathrm{C} 13$
$\mathrm{C} 22-\mathrm{N} 21-\mathrm{C} 26-\mathrm{C} 25$
$\mathrm{C} 22-\mathrm{C} 23-\mathrm{C} 24-\mathrm{C} 25$
$\mathrm{C} 24-\mathrm{C} 25-\mathrm{C} 26-\mathrm{N} 21$
$\mathrm{C} 24-\mathrm{C} 23-\mathrm{C} 22-\mathrm{N} 21$

109.4

109.4

111.05 (13)

109.4

109.4

109.6

109.6

$110.24(12)$

108.1

109.6

109.6

$-55.57(16)$

$57.09(17)$

$54.49(17)$

$-58.61(16)$

$-59.86(16)$

$57.84(16)$

$-55.39(18)$

$-55.37(16)$

56.89 (17)
N31- C36- H36B

C35-C $36-\mathrm{H} 36 \mathrm{~A}$

C $35-\mathrm{C} 36-\mathrm{H} 36 \mathrm{~B}$

H36A-C36-H36B

$\mathrm{C} 33-\mathrm{C} 34-\mathrm{C} 35$

C33-C34-H34A

C33-C $34-\mathrm{H} 34 \mathrm{~B}$

C $35-\mathrm{C} 34-\mathrm{H} 34 \mathrm{~A}$

C35-C34-H34B

$\mathrm{H} 34 \mathrm{~A}-\mathrm{C} 34-\mathrm{H} 34 \mathrm{~B}$

$\mathrm{C} 12-\mathrm{N} 11-\mathrm{C} 16-\mathrm{C} 15$

$\mathrm{C} 15-\mathrm{C} 14-\mathrm{C} 13-\mathrm{C} 12$

$\mathrm{C} 13-\mathrm{C} 14-\mathrm{C} 15-\mathrm{C} 16$

C32-N31-C36-C35

$\mathrm{C} 32-\mathrm{C} 33-\mathrm{C} 34-\mathrm{C} 35$

$\mathrm{C} 36-\mathrm{N} 31-\mathrm{C} 32-\mathrm{C} 33$

$\mathrm{C} 36-\mathrm{C} 35-\mathrm{C} 34-\mathrm{C} 33$

$\mathrm{C} 34-\mathrm{C} 33-\mathrm{C} 32-\mathrm{N} 31$

$\mathrm{C} 34-\mathrm{C} 35-\mathrm{C} 36-\mathrm{N} 31$
109.8

109.8

109.8

108.2

$109.69(13)$

109.7

109.7

109.7

109.7

108.2

$59.41(16)$

$-54.79(17)$

$53.79(17)$

$-59.33(17)$

$55.93(17)$

$59.01(17)$

$-55.78(18)$

$-57.02(17)$

57.05 (18)

Hydrogen-bond geometry $\left(A,{ }^{\circ}\right)$

\begin{tabular}{lllll}
\hline$D-\mathrm{H} \cdots A$ & $D-\mathrm{H}$ & $\mathrm{H} \cdots A$ & $D \cdots A$ & $D-\mathrm{H} \cdots A$ \\
\hline $\mathrm{O} 14-\mathrm{H} 14 \cdots \mathrm{O} 21$ & 0.84 & 1.72 & $2.5603(16)$ & 173 \\
$\mathrm{~N} 21-\mathrm{H} 21 A \cdots \mathrm{O} 11^{\mathrm{i}}$ & 0.91 & 1.93 & $2.8226(18)$ & 166 \\
$\mathrm{~N} 21-\mathrm{H} 21 B \cdots \mathrm{O} 12$ & 0.91 & 2.32 & $2.9096(19)$ & 122 \\
$\mathrm{~N} 21-\mathrm{H} 21 B \cdots \mathrm{O} 24^{\mathrm{ii}}$ & 0.91 & 2.47 & $3.0964(18)$ & 127 \\
$\mathrm{~N} 11-\mathrm{H} 11 A \cdots \mathrm{O} 21$ & 0.91 & 2.59 & $3.201(2)$ & 126 \\
$\mathrm{~N} 11-\mathrm{H} 11 A \cdots \mathrm{O} 24$ & 0.91 & 1.89 & $2.7904(17)$ & 171 \\
$\mathrm{~N} 11-\mathrm{H} 11 B \cdots \mathrm{O} 22^{\mathrm{iii}}$ & 0.91 & 2.47 & $3.0474(18)$ & 122 \\
$\mathrm{~N} 11-\mathrm{H} 11 B \cdots \mathrm{O} 23^{\mathrm{iii}}$ & 0.91 & 1.92 & $2.8039(18)$ & 164 \\
$\mathrm{~N} 31-\mathrm{H} 31 A \cdots \mathrm{O} 12$ & 0.91 & 2.41 & $3.0245(18)$ & 125 \\
$\mathrm{~N} 31-\mathrm{H} 31 A \cdots \mathrm{O} 13$ & 0.91 & 1.93 & $2.8240(18)$ & 167 \\
$\mathrm{~N} 31-\mathrm{H} 31 B \cdots \mathrm{O} 24^{\mathrm{ii}}$ & 0.91 & 1.89 & $2.7978(19)$ & 172
\end{tabular}

Symmetry codes: (i) $-x+1,-y+1,-z+1$; (ii) $x,-y+3 / 2, z+1 / 2$; (iii) $x,-y+3 / 2, z-1 / 2$. 